\title{
Existence of vector bundles and global resolutions for singular surfaces
}

\author{
Stefan Schröer and Gabriele Vezzosi
}

\begin{abstract}
We prove two results about vector bundles on singular algebraic surfaces. First, on proper surfaces there are vector bundles of rank two with arbitrarily large second Chern number and fixed determinant. Second, on separated normal surfaces any coherent sheaf is the quotient of a vector bundle. As a consequence, for such surfaces the Quillen $K$-theory of vector bundles coincides with the Waldhausen $K$-theory of perfect complexes. Examples show that, on non-separated schemes, usually many coherent sheaves are not quotients of vector bundles.
\end{abstract}

\section{Introduction}

Let $Y$ be a scheme. We pose the following question: Does there exist a non-trivial locally free $\mathcal{O}_{Y}$-module of finite rank? Many fundamental problems in algebraic geometry are related to this. We mention a few: In the theory of moduli spaces, one wants to know whether certain moduli spaces of semistable vector bundles with given Chern numbers are non-empty. In $K$-theory, one seeks to replace coherent sheaves by complexes of locally free sheaves, or perfect complexes by bounded complexes of locally free sheaves. In the theory of Brauer groups, a fundamental problem is to find a locally free sheaf admitting an Azumaya algebra structure with given cohomology class.

There is a good chance of tackling such problems if $Y$ admits an ample invertible sheaf, or more generally an ample family of invertible sheaves [Gro71, Exposé II]. Very few things, however, seem to be known in general. For example, it is open whether or not a proper algebraic scheme has non-trivial vector bundles at all.

The goal of this paper is to attack the problem for the simplest family of schemes where few results are known, namely algebraic surfaces with singularities over an arbitrary ground field. Our first main result (Theorem 1.1) is that a proper algebraic surface admits rank two vector bundles with arbitrary preassigned determinant and arbitrarily large second Chern number. The second main result (Theorem 2.1) is that, on normal separated algebraic surfaces, any coherent sheaf is the quotient of a locally free sheaf. In other words, any coherent sheaf admits a global resolution with locally free sheaves. Examples show that this fails for trivial reasons without the separatedness assumption (Proposition 4.2). Using Theorem 2.1 we infer that on normal separated surfaces Quillen's $K$-theory of vector bundles coincides with the Waldhausen $K$-theory of perfect complexes (Theorem 3.3).

The situation for algebraic surfaces with singularities is similar to the case of non-algebraic smooth compact complex surfaces. Schuster [Sch82] extended the existence of global resolution for a smooth algebraic surface to smooth compact complex surfaces. For non-algebraic smooth compact complex surfaces, Bănică and Le Potier [BLP87] proved that Chern numbers for torsion free sheaves

Received 20 March 2002, accepted in final form 7 May 2002.

2000 Mathematics Subject Classification 14F05, 14J60, $14 \mathrm{C} 35$.

Keywords: global resolutions, vector bundles, singular surfaces, $K$-groups.

This journal is (C) Foundation Compositio Mathematica 2004. 


\section{S. SChrÖER AND G. Vezzosi}

satisfy certain inequalities. For rank two vector bundles, this reduces to $2 c_{2} \geqslant c_{1}^{2}$, so the second Chern number is bounded from below if the determinant is fixed.

This analogy breaks down in higher dimensions. Burt Totaro pointed out to us recent results of Claire Voisin: Among other things, she proved that ideal sheaves of closed points on general complex tori of dimension $\geqslant 3$ are not quotients of locally free sheaves [Voi01, Corollary 2].

\section{Existence of vector bundles}

Throughout this paper we work over a fixed (but arbitrary) ground field $k$. In this section, $Y$ denotes a proper surface. The word surface means a $k$-scheme of finite type whose irreducible components are two-dimensional. We pose the following question: 'Given an invertible $\mathcal{O}_{Y}$-module $\mathcal{L}$ and an integer $c_{2} \in \mathbb{Z}$, does there exist a locally free $\mathcal{O}_{Y}$-module $\mathcal{F}$ of rank two with $\operatorname{determinant} \operatorname{det}(\mathcal{F}) \simeq \mathcal{L}$ and second Chern number $c_{2}(\mathcal{F})=c_{2}$ ?'

Schwarzenberger proved such a result for smooth surfaces over algebraically closed ground fields [Sch61, Theorem 8]. Beyond this, however, little seems to be known. It might easily happen that $\operatorname{Pic}(Y)=0$ for normal surfaces (see $[\operatorname{Sch} 99, \S 3]$ ), and it is a priori unclear whether or not there are any non-trivial vector bundles on arbitrary singular surfaces (though, by [Sch01, Theorem 4.1], any proper normal surface has non-trivial vector bundles of square rank).

To start with, let us briefly recall the notions of $S_{1}$-ization and $S_{2}$-ization since we will use them quite often. Given (a surface) $Y$, its $S_{1}$-ization is the closed subscheme $Y^{\prime} \subset Y$ having the same underlying topological space as $Y$ and whose defining ideal is the ideal $\mathcal{I} \subset \mathcal{O}_{Y}$ of sections whose support has dimension $\leqslant 1$. The $S_{2}$-ization $f: X \rightarrow Y$ of $Y$ is defined as follows: For any $y \in Y$, the stalk $f_{*}\left(\mathcal{O}_{X}\right)_{y}$ is the intersection of all local rings $\mathcal{O}_{Y^{\prime}, x}$ for all points $x \in \operatorname{Spec}\left(\mathcal{O}_{Y, y}\right)$ of codimension one, the intersection taking place in the ring of total fractions for $\mathcal{O}_{Y^{\prime}, y}$. According to [Gro65, Proposition 5.11.1], the $S_{2}$-ization $f: X \rightarrow Y$ is a finite birational morphism. Note that $Y^{\prime}$ and $X$ satisfy Serre's condition $\left(\mathrm{S}_{1}\right)$ and $\left(\mathrm{S}_{2}\right)$, respectively.

Next, it is perhaps a good idea to define for our situation what we mean by the second Chern number $c_{2}(\mathcal{F}) \in \mathbb{Z}$ for a given locally free $\mathcal{O}_{Y}$-module $\mathcal{F}$ of rank $r \geqslant 0$. The following naive approach suits us well. Let $f: X \rightarrow Y$ be the $S_{2}$-ization. To define $c_{2}(\mathcal{F})$, set $\mathcal{E}=f^{*}(\mathcal{F})$ and $\mathcal{L}=\operatorname{det}(\mathcal{E})$. The Riemann-Roch equation

$$
\chi(\mathcal{E})=\frac{1}{2} \mathcal{L} \cdot\left(\mathcal{L}-\omega_{X}\right)-c_{2}(\mathcal{E})+r \chi\left(\mathcal{O}_{X}\right)
$$

defines an integer $c_{2}(\mathcal{E})$, and we set $c_{2}(\mathcal{F})=c_{2}(\mathcal{E})$. Here $\omega_{X}$ is the dualizing sheaf, which exists because $X$ is Cohen-Macaulay.

We now state our first main result.

TheOREM 1.1. Let $Y$ be a proper surface. Then for each invertible $\mathcal{O}_{Y}$-module $\mathcal{L}$ and each integer $c_{2} \geqslant 0$, there is a locally free $\mathcal{O}_{Y}$-module $\mathcal{F}$ of rank two satisfying $\operatorname{det}(\mathcal{F}) \simeq \mathcal{L}$ and $c_{2}(\mathcal{F}) \geqslant c_{2}$.

The proof requires some preparation, in the form of three propositions. Suppose we have a proper birational morphism $f: X \rightarrow Y$. The union of all integral curves mapped to a point is called the exceptional curve $E \subset X$. The union of all integral curves given by the codimension one points $x \in X$ for which $\mathcal{O}_{Y, f(x)} \rightarrow \mathcal{O}_{X, x}$ is finite but not bijective is called the ramification curve. The following tells us that in constructing vector bundles, we may pass to certain auxiliary surfaces.

Proposition 1.2. Let $f: X \rightarrow Y$ be a proper birational morphism with empty ramification curve. Suppose the exceptional curve $E \subset X$ supports an effective Cartier divisor $D \subset X$ with $\mathcal{O}_{E}(-D)$ ample. Then for each $r \geqslant 0$, there is an $n \geqslant 1$ such that the functor $\mathcal{F} \mapsto f^{*}(\mathcal{F})$ induces a surjection from the set of isomorphism classes of locally free $\mathcal{O}_{Y}$-modules of rank $r$ to the set of isomorphism classes of locally free $\mathcal{O}_{X}$-modules of rank $r$ whose restriction to $n D$ is trivial. 


\section{VECTOR BUNDLES AND GLOBAL RESOLUTIONS}

Proof. First consider the special case that $f: X \rightarrow Y$ is finite. Then the exceptional curve $E \subset X$ is empty, and $\mathcal{O}_{Y} \rightarrow f_{*}\left(\mathcal{O}_{X}\right)$ is bijective outside finitely many closed points. Fix a locally free $\mathcal{O}_{X}$-module $\mathcal{E}$ of rank $r$. For each $y \in Y$, the preimage $f^{-1}\left(\operatorname{Spec} \mathcal{O}_{Y, y}\right)$ is a semilocal scheme, on which $\mathcal{E}$ becomes trivial. Consequently, there is an affine open covering $V_{i} \subset Y$ such that $\mathcal{E}$ becomes trivial on the preimages $U_{i}=f^{-1}\left(V_{i}\right)$. Set $\mathfrak{V}=\left(V_{i}\right)$ and $\mathfrak{U}=\left(U_{i}\right)$. Passing to a refinement of $\mathfrak{V}$, we may assume that each $V_{i}$ contains at most one point $y \in Y$ where $\mathcal{O}_{Y} \rightarrow f_{*}\left(\mathcal{O}_{X}\right)$ is not bijective. Then $U_{i} \cap U_{j}=V_{i} \cap V_{j}$ for $i \neq j$, hence the canonical map on alternating cocycles $Z^{1}\left(\mathfrak{V}, \mathrm{GL}_{r, Y}\right) \rightarrow Z^{1}\left(\mathfrak{U}, \mathrm{GL}_{r, X}\right)$ is bijective. It follows that the functor $\mathcal{F} \mapsto f^{*}(\mathcal{F})$ is surjective on isomorphism classes.

Now let $f: X \rightarrow Y$ be arbitrary. Applying the preceding special case to the Stein factorization $\operatorname{Spec}\left(f_{*} \mathcal{O}_{X}\right) \rightarrow Y$, we may replace $Y$ by $\operatorname{Spec}\left(f_{*} \mathcal{O}_{X}\right)$ and assume that $\mathcal{O}_{Y} \rightarrow f_{*}\left(\mathcal{O}_{X}\right)$ is bijective. By the Theorem on Formal Functions [Gro61, Theorem 4.1.5], the functor $\mathcal{F} \mapsto f^{*}(\mathcal{F})$ is an equivalence between the categories of locally free $\mathcal{O}_{Y}$-modules $\mathcal{F}$ of rank $r$ and the category of locally free $\mathcal{O}_{X^{-}}$ modules $\mathcal{E}$ of rank $r$ whose restriction to the formal completion $\mathfrak{X}=X_{/ E}$ is trivial. The same argument as in [Sch01, Lemma 2.2] shows that there is an integer $n \geqslant 1$ such that $\mathcal{E}$ is trivial on $\mathfrak{X}$ if and only if it is trivial on $n D$, and the result follows.

Note that [Sch01, Lemma 2.2] is stated for resolutions of singularities of normal surfaces, but the proof holds literally true in our situation. That result also involves a family $\mathcal{B}$ of locally free $\mathcal{O}_{E}$-modules that is bounded up to tensoring with line bundles; here we apply the result with the trivial family $\mathcal{B}=\left\{\mathcal{O}_{E}\right\}$.

Remark 1.3. If $\mathcal{O}_{Y} \rightarrow f_{*}\left(\mathcal{O}_{X}\right)$ is bijective, the preceding arguments show that the functor $\mathcal{F} \mapsto$ $f^{*}(\mathcal{F})$ yields a bijection between the set of isomorphism classes of locally free $\mathcal{O}_{Y}$-modules of rank $r$ and the set of isomorphism classes of locally free $\mathcal{O}_{X}$-modules of rank $r$ whose restriction to $n D$ is trivial.

Passing to such auxiliary surfaces $X$ does not change second Chern numbers:

Proposition 1.4. Let $f: X \rightarrow Y$ be a proper birational morphism, $R \subset X$ its ramification curve, $\mathcal{F}$ a locally free $\mathcal{O}_{Y}$-module of finite rank, and $\mathcal{E}=f^{*}(\mathcal{F})$ its pullback. If $\mathcal{F}$ is trivial on $f(R)$, then $c_{2}(\mathcal{F})=c_{2}(\mathcal{E})$.

Proof. Making base change with the $S_{1}$-ization of $Y$ and replacing $X$ by its $S_{2}$-ization, we may assume that $Y$ has no embedded components and that $X$ is Cohen-Macaulay. If $f: X \rightarrow Y$ is finite, it must factor over the $S_{2}$-ization of $Y$. If $\mathcal{O}_{Y} \rightarrow f_{*}\left(\mathcal{O}_{X}\right)$ is bijective, then $Y$ already satisfy Serre's condition $\left(\mathrm{S}_{2}\right)$. Using Stein factorization, we therefore may assume that $Y$ is Cohen-Macaulay.

The trace map $f_{*}\left(\omega_{X}\right) \rightarrow \omega_{Y}$ is bijective outside the union of $f(R)$ and an additional finite subset. Since $\mathcal{M}=\operatorname{det}(\mathcal{F})$ is trivial on this set, the projection formula gives $\mathcal{M} \cdot \omega_{Y}=f^{*}(\mathcal{M}) \cdot \omega_{X}$. Furthermore, we have $\chi(\mathcal{F})-\chi(\mathcal{E})=r \chi\left(\mathcal{O}_{Y}\right)-r \chi\left(\mathcal{O}_{X}\right)$, because $\mathcal{F}$ is trivial on $f(R)$. Now the Riemann-Roch formula $(1)$ immediately implies $c_{2}(\mathcal{F})=c_{2}(\mathcal{E})$.

Now we come to the construction of auxiliary surfaces.

Proposition 1.5. Let $Y$ be a proper surface. Then there is a projective birational morphism $f: X \rightarrow Y$ with empty ramification curve such that the following hold:

i) there is a Cartier divisor $D \subset X$ supported by the exceptional curve $E \subset X$ with $\mathcal{O}_{E}(-D)$ ample;

ii) there is an irreducible Cartier divisor $C \subset X$ contained in the Cohen-Macaulay locus of $X$ and having no component in common with $E \subset X$. 


\section{S. SCHRÖER AND G. Vezzosi}

Proof. Choose a codimension one point $y \in Y$ not contained in $\operatorname{Ass}\left(\mathcal{O}_{Y}\right)$ so that $Y_{\text {red }}$ is normal near $y \in Y_{\text {red. }}$. There is a regular element $s \in \mathfrak{m}_{Y, y}$ such that $\mathcal{O}_{Y, y} /(s)$ is zero-dimensional. Let $B \subset Y$ be the schematic image of the canonical morphism $\operatorname{Spec} \mathcal{O}_{Y, y} /(s) \rightarrow Y$. Then $B \subset Y$ is an irreducible curve and Cartier outside finitely many closed points.

We now make a sequence of blow-ups $Y_{4} \rightarrow \cdots \rightarrow Y_{1} \rightarrow Y$ whose centers are infinitesimal near to $B$ and have zero-dimensional image on $B$. We shall denote by $B_{i} \subset Y_{i}$ the strict transform of $B$. First, we blow up the intersections $B \cap \overline{\{y\}}$, where $y$ ranges over the non-generic associated points $y \in \operatorname{Ass}\left(\mathcal{O}_{X}\right)$. Then the strict transform $B_{1} \subset Y_{1}$ becomes disjoint from embedded components. Next, let $H_{1} \subset Y_{1}$ be the branch locus for the normalization map $Y_{1}^{\text {nor }} \rightarrow Y_{1}^{\text {red }}$, and $Y_{2} \rightarrow Y_{1}$ be the blow-up with center $H_{1} \cap B_{1}$. Write $R_{2} \subset Y_{2}$ for the exceptional curve of $Y_{2} \rightarrow Y_{1}$, and $H_{2}$ for the strict transform of $H_{1}$.

To define the next blow-up, we first choose an open subset $U_{2} \subset Y_{2}^{\text {red }}$ containing the support of $B_{2}$ and disjoint from $H_{2}$, so that $R_{2} \cup B_{2}$ contains the singular locus of $U_{2}$. Let $r: U_{3} \rightarrow U_{2}$ be a resolution of singularities. Then there is an effective Cartier divisor $R_{3} \subset U_{3}$ supported by $r^{-1}\left(\operatorname{Sing}\left(U_{2}\right)\right)$ such that $-R_{3}$ is relatively ample with respect to $r$. Consequently, $r_{*} \mathcal{O}_{U_{3}}\left(-n R_{3}\right) \subset$ $r_{*} \mathcal{O}_{U_{3}}$ is contained in $\mathcal{O}_{U_{2}}$ for $n \gg 0$, and it then follows as in the proof of [Gro61, Proposition 2.3.5] that $r: U_{3} \rightarrow U_{2}$ is the blow-up of some center $Z_{2} \subset \operatorname{Sing}\left(U_{2}\right)$. Now let $Y_{3} \rightarrow Y_{2}$ be the blow-up of the schematic closure $\overline{Z_{2}} \subset Y_{2}$. By construction, the strict transform $B_{4} \subset Y_{4}$ is supported by the regular locus of $Y_{4}^{\text {red }}$.

Let $g: Y^{\prime} \rightarrow Y$ be the composite morphism for the preceding sequence of blow-ups. According to [Ray71, Lemma 5.1.4], this is a blow-up of a zero-dimensional closed subscheme $Z \subset Y$ supported by $B$. The preimage $D^{\prime}=g^{-1}(Z)$ is an effective Cartier divisor supported on the exceptional curve, whose ideal is isomorphic to $\mathcal{O}_{Y^{\prime}}(1)$. In other words, it is ample on the exceptional curve.

Let $B^{\prime} \subset Y^{\prime}$ be the strict transform of $B$. Let $h: X \rightarrow Y^{\prime}$ be the blow-up of $B^{\prime}$. This is a homeomorphism, because $B^{\prime} \cap Y_{\text {red }}^{\prime} \subset Y_{\text {red }}^{\prime}$ is already a Cartier divisor. Consequently, the effective Cartier divisor $C=h^{-1}\left(B^{\prime}\right)$ has no irreducible component in common with the exceptional curve $E \subset X$ for the composition $f: X \rightarrow Y$. Replacing $X$ in some open neighborhood of $C$ by its $S_{2}$-ization, we may also assume that $C$ lies in the Cohen-Macaulay locus. Since $h$ is finite, the Cartier divisor $D=h^{-1}\left(D^{\prime}\right)$ has $\mathcal{O}_{E}(-D)$ ample.

Proof of Theorem 1.1. First choose a proper birational morphism $f: X \rightarrow Y$ and Cartier divisors $C \subset X$ and $D \subset X$ as in Proposition 1.5. Then choose an integer $n \geqslant 1$ as in Proposition 1.2 so that any locally free $\mathcal{O}_{X}$-module of rank two that is trivial on $n D$ comes from a locally free $\mathcal{O}_{Y}$-module of rank two.

In light of Proposition 1.4, we have to solve the following problem: Given an invertible $\mathcal{O}_{X^{-}}$ module $\mathcal{L}$ that is the preimage of an invertible $\mathcal{O}_{Y}$-module, find a locally free $\mathcal{O}_{X}$-module $\mathcal{E}$ of rank two whose restriction to $n D$ is trivial, so that $\operatorname{det}(\mathcal{E}) \simeq \mathcal{L}$ and $c_{2}(\mathcal{E}) \gg 0$. The idea is to start with $\mathcal{E}_{0}=\mathcal{L} \oplus \mathcal{O}_{X}$ and apply two elementary transformations (see [Mar82]) that cancel each other on $n D$. Note that $\operatorname{det}\left(\mathcal{E}_{0}\right)=\mathcal{L}$ and $c_{2}\left(\mathcal{E}_{0}\right)=0$.

To proceed, choose an ample effective Cartier divisor $A \subset C$ and an integer $t>0$ so that $H^{1}\left(C, \mathcal{L}_{C}((t-1) A)\right)=0$. Let $s_{1}: \mathcal{L}_{C} \rightarrow \mathcal{L}_{C}(t A)$ be the map induced by $t A$. The exact sequence

$$
H^{0}\left(C, \mathcal{L}_{C}(t A)\right) \longrightarrow H^{0}\left(A, \mathcal{L}_{A}(t A)\right) \longrightarrow H^{1}\left(C, \mathcal{L}_{C}((t-1) A)\right)
$$

shows that some map $s_{2}: \mathcal{O}_{C} \rightarrow \mathcal{L}_{C}(t A)$ has no zeros on $A$. This gives a surjection $s:\left.\mathcal{E}_{0}\right|_{C} \rightarrow$ $\mathcal{L}_{C}(t A)$. In turn, the exact sequence

$$
0 \longrightarrow \mathcal{E}_{1} \longrightarrow \mathcal{E}_{0} \longrightarrow \mathcal{L}_{C}(t A) \longrightarrow 0
$$

defines a coherent $\mathcal{O}_{X}$-module $\mathcal{E}_{1}$ of rank two, which is locally free because the stalks of $\mathcal{L}_{C}(t A)$ have projective dimension one. In other words, $\mathcal{E}_{1}$ is an elementary transformation of $\mathcal{E}_{0}$. 


\section{VECTOR BUNDLES AND GLOBAL RESOLUTIONS}

Taking determinants on $X-A$, we compute $\operatorname{det}\left(\mathcal{E}_{1}\right)=\mathcal{L}(-C)$. This works because $X$ satisfies Serre's condition $\left(\mathrm{S}_{2}\right)$ near $A$. Restricting the exact sequence (2) to $n D$, we obtain an exact sequence

$$
\left.\left.\mathcal{T}_{o r_{1}}\left(\mathcal{O}_{n D}, \mathcal{O}_{C}(t A)\right) \longrightarrow \mathcal{E}_{1}\right|_{n D} \longrightarrow \mathcal{E}_{0}\right|_{n D} \longrightarrow \mathcal{O}_{C \cap n D} \longrightarrow 0 .
$$

The map on the left vanishes: On the one hand, $\mathcal{T}_{\text {or }}\left(\mathcal{O}_{n D}, \mathcal{O}_{C}(t A)\right)$ is supported on $C \cap D$. On the other hand, $\left.\mathcal{E}_{1}\right|_{n D}$ has no torsion sections near $C \cap D$, because $D$ is Cartier and $C$ is contained in the $S_{2}$-locus of $X$. We conclude that the restriction $\left.\mathcal{E}_{1}\right|_{n D}$ remains an elementary transformation of $\left.\mathcal{E}_{0}\right|_{n D}$.

Next, consider the invertible $\mathcal{O}_{C}$-module $\mathcal{N}_{1}=\mathcal{E} x t^{1}\left(\mathcal{L}_{C}(t A), \mathcal{O}_{X}\right)$. Dualizing the exact sequence (2), we obtain an exact sequence

$$
0 \longrightarrow \mathcal{E}_{0}^{\vee} \longrightarrow \mathcal{E}_{1}^{\vee} \longrightarrow \mathcal{N}_{1} \longrightarrow 0 .
$$

Its restriction to $C$ gives an exact sequence

$$
\left.0 \longrightarrow \mathcal{N}_{2} \longrightarrow \mathcal{E}_{1}^{\vee}\right|_{C} \longrightarrow \mathcal{N}_{1} \longrightarrow 0
$$

for some invertible $\mathcal{O}_{C}$-module $\mathcal{N}_{2}$. Now choose an ample Cartier divisor $A^{\prime} \subset C$ disjoint from $D \cap C$. Choose $k_{0}>0$ so that we have $H^{1}\left(C, \mathcal{O}_{C}\left(k A^{\prime}-n D\right)\right)=0$ and $H^{1}\left(C, \mathcal{N}_{1} \otimes \mathcal{N}_{2}^{\vee}\left((k-1) A^{\prime}-n D\right)\right)=0$ for all $k \geqslant k_{0}$. Fix such an integer $k \geqslant k_{0}$, and let $s_{1}^{\prime}: \mathcal{N}_{1} \rightarrow \mathcal{N}_{1}\left(k A^{\prime}\right)$ be the canonical map given by $k A^{\prime}$. The exact sequence (4) yields an exact sequence

$$
H^{0}\left(C, \mathcal{N}_{2}^{\vee} \otimes \mathcal{N}_{1}\left(k A^{\prime}\right)\right) \longrightarrow H^{0}\left(C, \mathcal{O}_{A^{\prime}} \oplus \mathcal{O}_{C \cap n D}\right) \longrightarrow H^{1}\left(\mathcal{N}_{2}^{\vee} \otimes \mathcal{N}_{1}\left((k-1) A^{\prime}-n D\right)\right),
$$

showing that some map $s_{2}^{\prime}: \mathcal{N}_{2} \rightarrow \mathcal{N}_{1}\left(k A^{\prime}\right)$ has no zeros on $A^{\prime}$ and vanishes on $C \cap n D$. The exact sequence

$$
\operatorname{Hom}\left(\left.\mathcal{E}_{1}^{\vee}\right|_{C}, \mathcal{N}_{1}\left(k A^{\prime}-n D\right)\right) \rightarrow \operatorname{Hom}\left(\mathcal{N}_{2}, \mathcal{N}_{1}\left(k A^{\prime}-n D\right)\right) \rightarrow \operatorname{Ext}^{1}\left(\mathcal{N}_{1}, \mathcal{N}_{1}\left(k A^{\prime}-n D\right)\right)
$$

tells us that there is an extension of the map $s_{2}^{\prime}: \mathcal{N}_{2} \rightarrow \mathcal{N}_{1}\left(k A^{\prime}\right)$ to a mapping $s_{2}^{\prime}:\left.\mathcal{E}_{1}^{\vee}\right|_{C} \rightarrow \mathcal{N}_{1}\left(k A^{\prime}\right)$ vanishing on $C \cap n D$. Setting $s^{\prime}=s_{1}^{\prime}+s_{2}^{\prime}$, we obtain a surjective mapping $s^{\prime}:\left.\mathcal{E}_{1}^{\vee}\right|_{C} \rightarrow \mathcal{N}_{1}\left(k A^{\prime}\right)$. By construction, this surjection and the old surjection $\left.\mathcal{E}_{1}^{\vee}\right|_{C} \rightarrow \mathcal{N}_{1}$ are isomorphic on $C \cap n D$. Now the exact sequence

$$
0 \longrightarrow \mathcal{E}_{2} \longrightarrow \mathcal{E}_{1}^{\vee} \longrightarrow \mathcal{N}_{1}\left(k A^{\prime}\right) \longrightarrow 0
$$

defines a locally free $\mathcal{O}_{X}$-module $\mathcal{E}_{2}$. Set $\mathcal{E}=\mathcal{E}_{2}^{\vee}$. Taking determinants on $X-A^{\prime}$, we obtain $\operatorname{det}(\mathcal{E})=\mathcal{L}$. Using the Riemann-Roch formula (1) on the $S_{2}$-ization of $X$, we compute

$$
c_{2}(\mathcal{E})=c_{2}\left(\mathcal{E}_{1}\right)+C \cdot \operatorname{det}\left(\mathcal{E}_{1}\right)+k \operatorname{deg}\left(A^{\prime}\right)+\operatorname{deg}\left(\mathcal{N}_{1}\right),
$$

which becomes arbitrarily large for $k \gg 0$. Finally, restriction to $n D$ gives an exact sequence

$$
\left.\left.0 \longrightarrow \mathcal{E}^{\vee}\right|_{n D} \longrightarrow \mathcal{E}_{1}^{\vee}\right|_{n D} \longrightarrow \mathcal{O}_{C \cap n D} \longrightarrow 0 .
$$

By construction, the elementary transformations (3) and (5) are dual to each other, so $\left.\left.\mathcal{E}\right|_{n D} \simeq \mathcal{E}_{0}\right|_{n D}$ is trivial. Thus $\mathcal{E}$ is a locally free $\mathcal{O}_{X}$-module with the desired properties.

Remark 1.6. The preceding proof shows that we may increase $c_{2}(\mathcal{F})$ without changing the determinant by adding the number $\operatorname{deg}\left(A^{\prime}\right)>0$. If $Y$ is reduced and the ground field $k$ is algebraically closed, we may choose the Cartier divisor $C \subset X$ reduced and the ample Cartier divisor $A^{\prime} \subset C$ of degree one. In this case, there is a constant $c \geqslant 0$ such that all numbers $c_{2} \geqslant c$ occur as second Chern number of locally free $\mathcal{O}_{Y}$-modules of rank two with fixed determinant.

Remark 1.7. If $\mathcal{M}_{1}$ and $\mathcal{M}_{2}$ are two invertible $\mathcal{O}_{Y}$-modules, the locally free $\mathcal{O}_{Y}$-module $\mathcal{F}=$ $\mathcal{M}_{1} \oplus \mathcal{M}_{2}$ has $c_{2}(\mathcal{F})=\mathcal{M}_{1} \cdot \mathcal{M}_{2}$. If the intersection form on $\operatorname{Pic}(Y)$ is non-zero, this immediately implies that $c_{2}$ can be arbitrarily positive and negative. Moreover, if the intersection form has a positive eigenvalue, say $\mathcal{M} \cdot \mathcal{M}>0$, then $\mathcal{F}=\mathcal{M} \oplus \mathcal{M}^{\vee}$ satisfies $c_{1}(\mathcal{F})=0$ and $c_{2}(\mathcal{F})=-\mathcal{M} \cdot \mathcal{M}$, so $c_{2}$ can be arbitrarily negative with $c_{1}$ fixed. 


\section{S. SCHRÖER AND G. VEZzosI}

Theorem 1.1 is mainly a result about surfaces whose intersection form is trivial. However, the intersection form is usually trivial for singular surfaces (compare [Sch99] and [Har77, p. 232, Exercise 5.9]).

Remark 1.8. Bănică and Le Potier [BLP87, Theorem 3.1] proved $2 c_{2}(\mathcal{F}) \geqslant c_{1}^{2}(\mathcal{F})$ for locally free sheaves $\mathcal{F}$ of rank two on non-algebraic smooth compact complex surfaces. In light of this, we do not expect that, on badly singular proper algebraic surfaces, the second Chern numbers for vector bundles of rank two with fixed determinant might be arbitrarily negative.

\section{Existence of global resolutions}

Let $Y$ be a separated surface. According to the Nagata Embedding Theorem [Lut93, Theorem 3.2], we may embed $Y$ into a proper surface, and Theorem 1.1 tells us that there are many locally free $\mathcal{O}_{Y}$-modules. A natural question to ask is this: Given a coherent $\mathcal{O}_{Y}$-module $\mathcal{M}$, does there exist a locally free $\mathcal{O}_{Y}$-module $\mathcal{F}$ of finite rank, together with a surjection $\mathcal{F} \rightarrow \mathcal{M}$ ? This holds if $Y$ admits an ample invertible sheaf, or more generally an ample family of invertible sheaves [Gro71, Exposé II, Proposition 2.2.3]. Schuster solved the analogous problem for smooth compact complex surfaces [Sch82], which are not necessarily algebraic. We have the following result.

Theorem 2.1. Let $Y$ be a separated normal surface. Then any coherent $\mathcal{O}_{Y}$-module $\mathcal{M}$ is the quotient of a locally free $\mathcal{O}_{Y}$-module $\mathcal{F}$ of finite rank.

The idea for the proof is to construct a sheaf of 1 -syzygies $\mathcal{S}$ for $\mathcal{M}$, and then obtain $\mathcal{F}$ as an extension of $\mathcal{M}$ by $\mathcal{S}$. Similarly, we obtain the sheaf of 1 -syzygies as the extension of an ideal by a suitable locally free sheaf. The main problem is to choose these sheaves carefully so that the desired extensions, which always exist locally, also exist globally. We start with some preliminary reductions. Throughout, $Y$ denotes a normal surface.

Proposition 2.2. Let $\mathcal{M}$ be a coherent $\mathcal{O}_{Y}$-module. Then there are finitely many effective Weil divisors $D_{1}, \ldots, D_{j} \subset Y$ and a surjection $\bigoplus_{i=1}^{j} \mathcal{O}_{Y}\left(-D_{i}\right) \rightarrow \mathcal{M}$ so that the induced maps $\bigoplus_{i=1}^{j} \mathcal{O}_{Y}$ $\left(-t D_{i}\right) \rightarrow \mathcal{M}$ remain surjective for all $t \geqslant 1$.

Proof. Since $\mathcal{M}$ is coherent, it suffices to see that each germ $s_{y} \in \mathcal{M}_{y}, y \in Y$, lies in the image of a map $\mathcal{O}_{Y}(-D) \rightarrow \mathcal{M}$ for some effective Weil divisor $D \subset Y$ not containing $y$. Choose a representative $s_{V} \in \Gamma(V, \mathcal{M})$ on some affine open subset $V \subset Y$. Since $Y$ is separated, the reduced complement $D=Y-U$ is an effective Weil divisor, say with ideal $\mathcal{I}=\mathcal{O}_{Y}(-D)$ [Gro67, Corollary 21.12.7]. According to [GD70, Proposition 6.9.17], we may extend $s_{V}: \mathcal{O}_{V} \rightarrow \mathcal{M}_{V}$ to a map $s: \mathcal{I}^{m} \rightarrow \mathcal{M}$ for certain $m \geqslant 1$. Now choose some $n \geqslant 1$ so that $n D$ contains $\operatorname{Spec}\left(\mathcal{O}_{Y} / \mathcal{I}^{m}\right)$. Then $\mathcal{O}_{Y}(-n D) \subset \mathcal{I}^{m}$, and the composition $\mathcal{O}_{Y}(-n D) \rightarrow \mathcal{M}$ is the desired map.

Next we reduce to the case that our ground field $k$ is infinite.

Proposition 2.3. Let $k \subset k^{s}$ be a separable closure, and $Y^{s}=Y \otimes k^{s}$ the induced normal surface. If all coherent $\mathcal{O}_{Y^{s}}$-modules are quotients of locally free $\mathcal{O}_{Y^{s}}$-modules of finite rank, then the same holds for all coherent $\mathcal{O}_{Y}$-modules.

Proof. Let $\mathcal{M}$ be a coherent $\mathcal{O}_{Y}$-module. Fix a germ $s_{y} \in \mathcal{M}_{y}, y \in Y$. As in the proof of Proposition 2.2, there is an effective Weil divisor $D \subset Y$ not containing $y$ and a map $\mathcal{O}_{Y}(-D) \rightarrow \mathcal{M}$ so that the stalk $s_{y}$ lies in the image. According to [Gro66, Theorem 8.5.2], there is a finite separable field extension $k \subset k^{\prime}$ so that on the normal surface $Y^{\prime}=Y \otimes k^{\prime}$, there is a locally free $\mathcal{O}_{Y^{\prime}}$-module $\mathcal{F}^{\prime}$ and a surjection $\mathcal{F}^{\prime} \rightarrow \mathcal{O}_{Y^{\prime}}\left(-D^{\prime}\right)$, where $D^{\prime}=D \otimes k^{\prime}$ is the induced Weil divisor. Dualizing, we obtain an injection $f^{*}\left(\mathcal{O}_{Y}(D)\right) \subset \mathcal{F}^{\vee}$ which is a direct summand near $f^{-1}(y)$, where $f: Y^{\prime} \rightarrow Y$ 


\section{VECTOR BUNDLES AND GLOBAL RESOLUTIONS}

denotes the canonical projection. This injection corresponds to an injective map $\mathcal{O}_{Y}(D) \rightarrow f_{*}\left(\mathcal{F}^{\prime \vee}\right)$, which is a direct summand near $y$. Setting $\mathcal{F}=f_{*}\left(\mathcal{F}^{\vee}\right)^{\vee}$, we obtain a map $\mathcal{F} \rightarrow \mathcal{O}_{Y}(-D)$ which is surjective near $y$. The image of the composite map $\mathcal{F} \rightarrow \mathcal{M}$ contains the germ $s_{y}$, and we infer that $\mathcal{M}$ is the quotient of a locally free $\mathcal{O}_{Y}$-module of finite rank.

The following fact from commutative algebra will be useful.

Proposition 2.4. Let $\mathcal{I}$ and $\mathcal{N}$ be coherent $\mathcal{O}_{Y}$-modules. If $\mathcal{N}$ is reflexive, then the canonical map $\mathcal{H o m}\left(\mathcal{I}^{\vee \vee}, \mathcal{N}\right) \rightarrow \mathcal{H o m}(\mathcal{I}, \mathcal{N})$ is bijective.

Proof. This is a local problem, so let us assume that $Y=\operatorname{Spec}(A)$ is affine. Set $I=\Gamma(Y, \mathcal{I})$ and $N=\Gamma(Y, \mathcal{N})$. According to [Har94, Proposition 1.7], there is an exact sequence $0 \rightarrow N \rightarrow L \rightarrow L^{\prime}$ for certain free $A$-modules $L$ and $L^{\prime}$. This gives an a commutative diagram with exact rows:

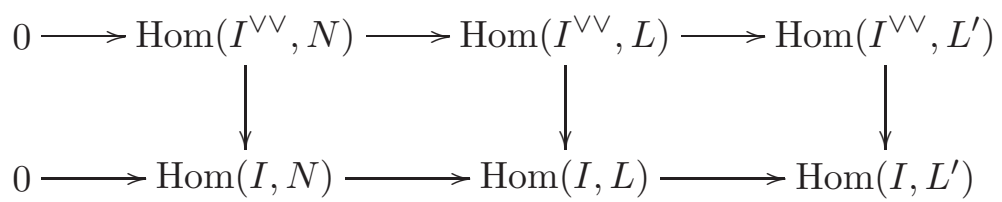

By the 5-Lemma, it suffices to treat the case $N=A$. Then our map in question becomes the canonical map $\left(I^{\vee \vee}\right)^{\vee} \rightarrow I^{\vee}$. By [Har94, Corollary 1.6], both $\left(I^{\vee \vee}\right)^{\vee}$ and $I^{\vee}$ are reflexive. At each prime ideal $\mathfrak{p} \subset A$ of height one, the module $I_{\mathfrak{p}}$ is the direct sum of a free module and a torsion module. Hence our map is bijective in codimension one, and [Har94, Theorem 1.12] implies that it is bijective everywhere.

Let us recall the notion of syzygies. Let $\mathcal{M}$ be a coherent $\mathcal{O}_{Y}$-module. A coherent $\mathcal{O}_{Y}$-module $\mathcal{S}$ is called a sheaf of 1-syzygies for $\mathcal{M}$ if, for any point $y \in Y$, there is an integer $m \geqslant 0$ and an exact sequence $0 \rightarrow \mathcal{S}_{y} \rightarrow \mathcal{O}_{Y, y}^{\oplus m} \rightarrow \mathcal{M}_{y} \rightarrow 0$. Our strategy to construct a surjection $\mathcal{F} \rightarrow \mathcal{M}$ from a locally free sheaf $\mathcal{F}$ of finite rank is first to prove the existence of a suitable sheaf of 1 -syzygies $\mathcal{S}$, and then to prove the existence of an extension $0 \rightarrow \mathcal{S} \rightarrow \mathcal{F} \rightarrow \mathcal{M} \rightarrow 0$ globalizing the local extensions. We now have all means to carry out this plan.

We are now in a position to return to the proof of Theorem 2.1.

Proof of Theorem 2.1. By the Nagata Embedding Theorem [Lut93, Theorem 3.2], we may embed $Y$ into a proper surface, because $Y$ is separated. Moreover, any coherent sheaf on $Y$ is the restriction of a coherent sheaf on the compactification by [GD70, Corollary 6.9.11]. So let us assume that $Y$ is proper. By Proposition 2.3, we may also assume that the ground field $k$ is separably closed, hence infinite.

Let $\mathcal{M}$ be a coherent $\mathcal{O}_{Y}$-module. We seek a locally free $\mathcal{O}_{Y}$-module $\mathcal{F}$ of finite rank, together with a surjection $\mathcal{F} \rightarrow \mathcal{M}$. In light of Proposition 2.2, we may assume that $\mathcal{M}=\mathcal{O}_{Y}(-D)$ for some effective Weil divisor $D \subset Y$. Moreover, we may replace $D$ by some high multiple and assume that $K_{Y}-2 D$ is not linearly equivalent to an effective Weil divisor.

Choose an integer $r \geqslant 2$ so that we have for each $y \in \operatorname{Sing}(Y)$ a surjection $\mathcal{O}_{Y, y}^{\oplus r+2} \rightarrow \mathcal{O}_{Y, y}(-D)$. The exact sequence

$$
0 \longrightarrow \mathcal{S}_{y} \longrightarrow \mathcal{O}_{Y, y}^{\oplus r+2} \longrightarrow \mathcal{O}_{Y, y}(-D) \longrightarrow 0
$$

defines $\mathcal{O}_{Y, y}$-modules $\mathcal{S}_{y}$ of rank $r+1$. Our first task is to extend these local modules to a coherent $\mathcal{O}_{Y}$-module $\mathcal{S}$ that is locally free on the regular locus. Then $\mathcal{S}$ would be a sheaf of 1 -syzygies for $\mathcal{M}=\mathcal{O}_{Y}(-D)$.

According to [EG85, Theorem 2.14], for each $y \in \operatorname{Sing}(Y)$ there is an ideal $\mathcal{I}_{y} \subset \mathcal{O}_{Y, y}$ and an exact sequence

$$
0 \longrightarrow \mathcal{O}_{Y, y}^{\oplus r} \longrightarrow \mathcal{S}_{y} \longrightarrow \mathcal{I}_{y} \longrightarrow 0
$$




\section{S. SCHRÖER AND G. Vezzosi}

Taking determinants on $\operatorname{Spec}\left(\mathcal{O}_{Y, y}\right)-\{y\}$, we see that $\mathcal{I}_{y}$ is isomorphic to $\mathcal{O}_{Y, y}(D)$ outside $y$. Hence there are isomorphisms $\mathcal{I}_{y}^{\vee \vee} \rightarrow \mathcal{O}_{Y, y}(D)$, and the cokernel $\mathcal{I}_{y}$ for the composite map $\mathcal{I}_{y} \rightarrow \mathcal{I}_{y}^{\vee \vee} \rightarrow$ $\mathcal{O}_{Y, y}(D)$ is supported by $y \in \operatorname{Spec}\left(\mathcal{O}_{Y, y}\right)$. Then $\mathcal{T}=\oplus \mathcal{T}_{y}$ is a skyscraper sheaf supported by $\operatorname{Sing}(Y)$, and we have a global surjection $\mathcal{O}_{Y}(D) \rightarrow \mathcal{T}$. Its kernel $\mathcal{I} \subset \mathcal{O}_{Y}(D)$ is a coherent fractional $\mathcal{O}_{Y}$-ideal extending the given local $\mathcal{O}_{Y, y}$-modules $\mathcal{I}_{y}$.

Suppose we have a locally free $\mathcal{O}_{Y}$-module $\mathcal{H}$ of $\operatorname{rank} r$. Then $\mathcal{E} x t^{1}(\mathcal{I}, \mathcal{H})$ is a coherent $\mathcal{O}_{Y}$-module supported on $\operatorname{Sing}(Y)$. Choose trivializations $\mathcal{H}_{y} \simeq \mathcal{O}_{Y, y}^{\oplus r}$. Then the local extensions $(7)$ correspond to a section $e \in H^{0}\left(Y, \mathcal{E} x t^{1}(\mathcal{I}, \mathcal{H})\right)$. The spectral sequence $H^{p}\left(Y, \mathcal{E} x t^{q}(\mathcal{I}, \mathcal{H})\right) \Rightarrow \operatorname{Ext}^{p+q}(\mathcal{I}, \mathcal{H})$ gives an exact sequence

$$
\operatorname{Ext}^{1}(\mathcal{I}, \mathcal{H}) \longrightarrow H^{0}\left(Y, \mathcal{E} x t^{1}(\mathcal{I}, \mathcal{H})\right) \longrightarrow H^{2}(Y, \mathcal{H o m}(\mathcal{I}, \mathcal{H}))
$$

We want to choose the locally free $\mathcal{O}_{Y}$-module $\mathcal{H}$ so that the term on the right vanishes. For then the section $e \in H^{0}\left(Y, \mathcal{E} x t^{1}(\mathcal{I}, \mathcal{H})\right)$ comes from a global extension $0 \rightarrow \mathcal{H} \rightarrow \mathcal{S} \rightarrow \mathcal{I} \rightarrow 0$, and we have constructed a sheaf of 1 -syzygies $\mathcal{S}$ for $\mathcal{M}$. The canonical map $\mathcal{H o m}\left(\mathcal{O}_{Y}(D), \mathcal{H}\right) \rightarrow \mathcal{H o m}(\mathcal{I}, \mathcal{H})$ is bijective by Proposition 2.4. Serre duality gives $H^{2}\left(Y, \mathcal{H o m}\left(\mathcal{O}_{Y}(D), \mathcal{H}\right)\right) \simeq H^{0}\left(Y, \mathcal{H}^{\vee} \otimes \omega_{Y}(D)\right)^{\vee}$. So we seek a locally free $\mathcal{O}_{Y}$-module $\mathcal{H}$ of rank $r$ without non-zero maps $\mathcal{H} \rightarrow \omega_{Y}(D)$.

To achieve this, let $f: X \rightarrow Y$ be a resolution of singularities, and $E \subset X$ the exceptional curve. According to Remark 1.3, there is an integer $n \geqslant 1$ such that the locally free $\mathcal{O}_{Y}$-modules of rank $r$ correspond to the locally free $\mathcal{O}_{X}$-modules of rank $r$ with trivial restriction to $n E$. Choose a Weil divisor $C$ on $X$ with $\mathcal{O}_{X}(C) \simeq f^{*}\left(\omega_{Y}(D)\right)^{\vee \vee}$.

Being regular, the proper surface $X$ is projective [Har70, Theorem 4.2]. Choose a very ample invertible $\mathcal{O}_{X}$-module $\mathcal{L}$ satisfying both $H^{2}\left(X, \mathcal{L}\left(K_{X}-C\right)\right)=0$ and $H^{1}\left(X, \mathcal{L}^{\otimes r}(-n E)\right)=0$. Consider the locally free $\mathcal{O}_{X}$-module $\mathcal{E}_{0}=\mathcal{L}^{\oplus r}$ of rank $r$. Then $\operatorname{Hom}\left(\mathcal{E}_{0}, \mathcal{O}_{X}(C)\right)=0$. The restriction $\left.\mathcal{E}_{0}\right|_{n E}$ is globally generated. Set $\Gamma=H^{0}\left(n E,\left.\mathcal{E}_{0}\right|_{n E}\right)$. The canonical surjection $\left.\Gamma \otimes \mathcal{O}_{n E} \rightarrow \mathcal{E}_{0}\right|_{n E}$ yields a morphism $\varphi: n E \rightarrow \operatorname{Grass}_{r}(\Gamma)$ into the Grassmannian of $r$-dimensional quotients. Choose a generic $r$-dimensional subvector space $\Gamma^{\prime} \subset \Gamma$. Here we use that our ground field is infinite. For each integer $k \geqslant 0$, let $G_{k} \subset \operatorname{Grass}_{r}(\Gamma)$ be the subscheme of surjections $\Gamma \rightarrow \Gamma^{\prime \prime}$ such that the composition $\Gamma^{\prime} \rightarrow \Gamma^{\prime \prime}$ has rank $\leqslant k$. Note that $G_{r-1}$ is a reduced Cartier divisor, and that $G_{r-2}$ has codimension four (see [ACG85, Section II.2]).

By the dimensional part of Kleiman's Transversality Theorem [Kle74, Theorem 2], the map $\varphi: n E \rightarrow \operatorname{Grass}_{r}(\Gamma)$ is disjoint to $G_{r-2}$ and passes through $G_{r-1}$ in finitely many points. The upshot of this is that the quotient of the canonical map $\left.\Gamma^{\prime} \otimes \mathcal{O}_{n E} \rightarrow \mathcal{E}_{0}\right|_{n E}$ is an invertible sheaf on some Cartier divisor $A \subset n E$. Consequently, we have constructed an exact sequence

$$
\left.0 \longrightarrow \mathcal{O}_{n E}^{\oplus r} \longrightarrow \mathcal{E}_{0}\right|_{n E} \longrightarrow \mathcal{O}_{A} \longrightarrow 0
$$

and $\mathcal{L}_{n E}^{\otimes r} \simeq \mathcal{O}_{n E}(A)$. The exact sequence

$$
H^{0}\left(X, \mathcal{L}^{\otimes r}\right) \longrightarrow H^{0}\left(n E, \mathcal{L}_{n E}^{\otimes r}\right) \longrightarrow H^{1}\left(X, \mathcal{L}^{\otimes r}(-n E)\right)
$$

shows that $A=H \cap n E$ for some divisor $H \subset X$ with $\mathcal{L}^{\otimes r} \simeq \mathcal{O}_{X}(H)$. The exact sequence

$$
H^{0}\left(H, \mathcal{E}_{0}^{\vee} \otimes \mathcal{N}\right) \longrightarrow H^{0}\left(A, \mathcal{E}_{0}^{\vee} \otimes \mathcal{N}_{A}\right) \longrightarrow H^{1}\left(H, \mathcal{E}_{0}^{\vee} \otimes \mathcal{N}(-A)\right)
$$

tells us that the surjection $\left.\mathcal{E}_{0}\right|_{n E} \rightarrow \mathcal{O}_{A}$ extends to a surjection $\mathcal{E}_{0} \rightarrow \mathcal{N}$ for any sufficiently ample invertible $\mathcal{O}_{H}$-module $\mathcal{N}$. Then the kernel $\mathcal{E} \subset \mathcal{E}_{0}$ is a locally free $\mathcal{O}_{X}$-module of rank $r$ that is trivial on $n E$, hence $\mathcal{H}=f_{*}(\mathcal{E})$ is locally free of rank $r$ as well.

Suppose there is a non-zero map $\mathcal{H} \rightarrow \omega_{Y}(D)$. Then there is also a non-zero map $\mathcal{E} \rightarrow \mathcal{O}_{X}(C)$. To rule this out, consider the exact sequence

$$
\operatorname{Hom}\left(\mathcal{E}_{0}, \mathcal{O}_{X}(C)\right) \longrightarrow \operatorname{Hom}\left(\mathcal{E}, \mathcal{O}_{X}(C)\right) \longrightarrow \operatorname{Ext}^{1}\left(\mathcal{N}, \mathcal{O}_{X}(C)\right)
$$




\section{VECTOR BUNDLES AND GLOBAL RESOLUTIONS}

The term on the right sits in an exact sequence

$$
H^{1}\left(X, \mathcal{H o m}\left(\mathcal{N}, \mathcal{O}_{X}(C)\right)\right) \longrightarrow \operatorname{Ext}^{1}\left(\mathcal{N}, \mathcal{O}_{X}(C)\right) \longrightarrow H^{0}\left(Y, \mathcal{E} x t^{1}\left(\mathcal{N}, \mathcal{O}_{X}(C)\right)\right) .
$$

We have $\operatorname{Hom}\left(\mathcal{N}, \mathcal{O}_{X}(C)\right)=0$ and $\mathcal{E} x t^{1}\left(\mathcal{N}, \mathcal{O}_{X}(C)\right) \simeq \mathcal{N}^{\vee}(C+H)$, which has no global sections if $\mathcal{N}$ is sufficiently ample. In other words, we may choose $\mathcal{N}$ so that $\operatorname{Hom}\left(\mathcal{H}, \omega_{Y}(D)\right)=0$. In turn, the desired global extension

$$
0 \longrightarrow \mathcal{H} \longrightarrow \mathcal{S} \longrightarrow \mathcal{I} \longrightarrow 0
$$

exists, and $\mathcal{S}$ is a sheaf of 1 -syzygies for $\mathcal{M}=\mathcal{O}_{Y}(-D)$.

Now we are almost done. The coherent $\mathcal{O}_{Y}$-module $\mathcal{E} x t^{1}(\mathcal{M}, \mathcal{S})$ is a skyscraper sheaf supported by $\operatorname{Sing}(Y)$. The local extensions from (6) define a global section $e^{\prime} \in H^{0}\left(X, \mathcal{E} x t^{1}(\mathcal{M}, \mathcal{S})\right)$. As above, we have an exact sequence

$$
\operatorname{Ext}^{1}(\mathcal{M}, \mathcal{S}) \longrightarrow H^{0}\left(Y, \mathcal{E} x t^{1}(\mathcal{M}, \mathcal{S})\right) \longrightarrow H^{2}(Y, \mathcal{H o m}(\mathcal{M}, \mathcal{S})) .
$$

We claim that the term on the right vanishes. Indeed, the canonical mapping $\mathcal{S} \rightarrow \mathcal{S}^{\prime}$ into the bidual $\mathcal{S}^{\prime}=\mathcal{S}^{\vee \vee}$ is bijective outside $\operatorname{Sing}(Y)$, so the induced map $H^{2}(Y, \mathcal{H o m}(\mathcal{M}, \mathcal{S})) \rightarrow H^{2}(Y, \mathcal{H}$ om $(\mathcal{M}$, $\left.\mathcal{S}^{\prime}\right)$ ) is bijective as well. Serre duality tells us that $H^{2}\left(Y, \mathcal{H o m}\left(\mathcal{M}, \mathcal{S}^{\prime}\right)\right) \simeq \operatorname{Hom}\left(\mathcal{S}^{\prime}, \omega_{Y}(-D)\right)^{\vee}$.

The exact sequence

$$
0 \longrightarrow \mathcal{H} \longrightarrow \mathcal{S}^{\prime} \longrightarrow \mathcal{O}_{Y}(D) \longrightarrow 0
$$

gives an exact sequence

$$
\operatorname{Hom}\left(\mathcal{O}_{Y}(D), \omega_{Y}(-D)\right) \longrightarrow \operatorname{Hom}\left(\mathcal{S}^{\prime}, \omega_{Y}(-D)\right) \longrightarrow \operatorname{Hom}\left(\mathcal{H}, \omega_{Y}(-D)\right) .
$$

The term on the right vanishes, because $\operatorname{Hom}\left(\mathcal{H}, \omega_{Y}(-D)\right)=0$ by construction of $\mathcal{H}$. The term on the left vanishes as well, because we have chosen the Weil divisor $D \subset Y$ from the very beginning so that $H^{0}\left(Y, \omega_{Y}(-2 D)\right)=0$. Summing up, the section $e^{\prime} \in H^{0}\left(Y, \mathcal{E} x t^{1}(\mathcal{M}, \mathcal{S})\right)$ comes from a global extension

$$
0 \longrightarrow \mathcal{S} \longrightarrow \mathcal{F} \longrightarrow \mathcal{M} \longrightarrow 0,
$$

where $\mathcal{F}$ is the desired locally free $\mathcal{O}_{Y}$-module of rank $r+2$.

The following application of Theorem 2.1 will be useful later.

Corollary 2.5. Let $Y$ be a normal separated surface. Then the following hold:

i) any quasicoherent $\mathcal{O}_{Y}$-module is a quotient of a locally free $\mathcal{O}_{Y}$-module of the form $\bigoplus_{\lambda \in L} \mathcal{F}_{\lambda}$ with each $\mathcal{F}_{\lambda}$ locally free of finite rank and $L$ possibly infinite;

ii) if $\mathcal{M} \rightarrow \mathcal{M}^{\prime}$ is a surjection of quasicoherent $\mathcal{O}_{Y}$-modules with $\mathcal{M}^{\prime}$ coherent, then there exists a locally free $\mathcal{O}_{Y}$-module of finite rank $\mathcal{F}$ and a homomorphism $\mathcal{F} \rightarrow \mathcal{M}$ such that the composition $\mathcal{F} \rightarrow \mathcal{M}^{\prime}$ remains surjective.

Proof.

i) For each coherent submodule $\mathcal{M}_{\lambda} \subset \mathcal{M}$ of a given quasicoherent $\mathcal{O}_{Y}$-module $\mathcal{M}$, choose a surjection $\mathcal{F}_{\lambda} \rightarrow \mathcal{M}_{\lambda}$ from a locally free sheaf of finite rank. The induced map $\bigoplus_{\lambda \in L} \mathcal{F}_{\lambda} \rightarrow \mathcal{M}$ is surjective, because $\mathcal{M}$ is the union of the $\mathcal{M}_{\lambda}$ by [GD70, Corollary 6.9.9].

ii) Choose a surjection $\bigoplus_{\lambda \in L} \mathcal{F}_{\lambda} \rightarrow \mathcal{M}$ as in (i). Since $\mathcal{M}^{\prime}$ is coherent and $Y$ is quasicompact, there is a finite subset $L^{\prime} \subset L$ such that $\mathcal{F}=\bigoplus_{\lambda \in L^{\prime}} \mathcal{F}_{\lambda}$ surjects onto $\mathcal{M}^{\prime}$.

\section{Applications to $K$-groups}

In this section we collect some more or less obvious applications of Theorem 2.1. Throughout, $Y$ denotes a normal separated surface. We start with the following proposition. 


\section{S. SChrÖER AND G. Vezzosi}

Proposition 3.1. Let $\mathcal{M}$ be a coherent $\mathcal{O}_{Y}$-module whose stalks have finite projective dimension. Then there is an exact sequence $0 \rightarrow \mathcal{F}_{2} \rightarrow \mathcal{F}_{1} \rightarrow \mathcal{F}_{0} \rightarrow \mathcal{M} \rightarrow 0$ where $\mathcal{F}_{0}, \mathcal{F}_{1}, \mathcal{F}_{2}$ are locally free $\mathcal{O}_{Y}$-modules of finite rank.

Proof. By the Auslander-Buchsbaum Formula [Eis95, Theorem 19.9], the stalks of $\mathcal{M}$ have projective dimension $\operatorname{pd}\left(\mathcal{M}_{y}\right) \leqslant \operatorname{depth}\left(\mathcal{O}_{Y, y}\right) \leqslant 2$. According to Theorem 2.1, we have an exact sequence $\mathcal{F}_{1} \rightarrow \mathcal{F}_{0} \rightarrow \mathcal{M} \rightarrow 0$ with $\mathcal{F}_{0}$ and $\mathcal{F}_{1}$ locally free of finite rank. Then the stalks of the kernel $\mathcal{F}_{2}$ for $\mathcal{F}_{1} \rightarrow \mathcal{F}_{0}$ have projective dimension $\leqslant 0$, hence $\mathcal{F}_{2}$ is locally free.

We come to $K$-theory. Let $W_{\text {perf }}(Y)$ be the additive category of perfect complexes of quasicoherent $\mathcal{O}_{Y}$-modules, and $W_{\text {naive }}(Y)$ be the additive category of bounded complexes of locally free $\mathcal{O}_{Y}$-modules. We obtain the corresponding derived categories $D_{\text {perf }}(Y)$ and $D_{\text {naive }}(Y)$ by inverting the quasi-isomorphisms.

Proposition 3.2. The natural inclusion $W_{\text {naive }}(Y) \subset W_{\text {perf }}(Y)$ induces an equivalence of triangulated categories $D_{\text {naive }}(Y) \simeq D_{\text {perf }}(Y)$.

Proof. We shall apply Illusie's result [Gro71, Exposé II, Proposition 1.2(c)]. In accordance with Illusie's notation, let $\underline{S}$ be the Zariski site of $Y, S:=Y, \underline{C}$ be the fibered category (over $\underline{S}$ ) of quasicoherent $\mathcal{O}$-modules, and $\underline{C}_{0}$ the fibered subcategory of locally free $\mathcal{O}$-modules of finite rank. Illusie's result has four assumptions, which take the following form.

First, the kernel of a surjection between locally free sheaves of finite rank is locally free of finite rank (' $\underline{C}_{0}$ est localement stable par noyau d'epimorphisme'), which is clearly true. Second, any surjection from a quasicoherent sheaf to a locally free sheaf of finite rank admits locally a section (' $\underline{C}_{0}$ est localement relevable'), which is also true. Third, if $\mathcal{M} \rightarrow \mathcal{M}^{\prime}$ is a surjection of quasicoherent sheaves on $Y$, with $\mathcal{M}^{\prime}$ locally free of finite rank, there is a map from a locally free sheaf of finite rank $\mathcal{F} \rightarrow \mathcal{M}$ such that $\mathcal{F} \rightarrow \mathcal{M}^{\prime}$ is surjective (' $\underline{C}_{0 Y}$ est quasi-relevable'), which holds by Corollary 2.5. Fourth, any coherent sheaf on $Y$ is the quotient of a locally free sheaf of finite rank ('tout objet de $\underline{C}_{Y}$ qui est de $\underline{C}_{0}$-type fini est de $\underline{C}_{0 Y}$-type fini'), which is Theorem 2.1.

Hence Illusie's result applies, and tells us that $D_{\text {naive }}(Y) \rightarrow D_{\text {perf }}(Y)$ is an equivalence of categories.

The category of perfect complexes $W_{\text {perf }}(Y)$ carries, in a canonical way, the structure of a complicial bi-Waldhausen category, as explained in [TT90, $\S 1]$. This additional structure gives rise to the Waldhausen $K$-theory spectrum $\mathbb{K}^{\text {perf }}(Y)$ and Waldhausen $K$-groups $K_{n}^{\text {perf }}(Y)=\pi_{n}\left(\mathbb{K}^{\text {perf }}(Y)\right)$. We also have the Quillen $K$-theory spectrum $\mathbb{K}^{\mathrm{Q}}(Y)$ and Quillen $K$-groups $K_{n}^{\mathrm{Q}}(Y)$, defined via the exact category of locally free $\mathcal{O}_{Y}$-modules of finite rank. These two approaches give the same result, up to homotopy:

Theorem 3.3. The natural map of spectra $\mathbb{K}^{\mathrm{Q}}(Y) \rightarrow \mathbb{K}^{\text {perf }}(Y)$ is a homotopy equivalence. In particular, the induced maps of groups $K_{n}^{\mathrm{Q}}(Y) \rightarrow K_{n}^{\text {perf }}(Y)$ are bijective for all $n \geqslant 0$.

Proof. Let $\mathbb{K}^{\text {naive }}(Y)$ be the Waldhausen $K$-theory spectrum for the complicial bi-Waldhausen category $W_{\text {naive }}(Y)$. By [TT90, Proposition 3.10], there is a canonical homotopy equivalence $\mathbb{K}^{\mathrm{Q}}(Y) \rightarrow$ $\mathbb{K}^{\text {naive }}(Y)$. This holds for arbitrary schemes. By [TT90, Theorem 1.9.8], together with Proposition 3.2 , the map $\mathbb{K}^{\text {naive }}(Y) \rightarrow \mathbb{K}^{\text {perf }}(Y)$ is a homotopy equivalence as well.

The group of connected components for $\mathbb{K}^{\mathrm{Q}}(X)$ is nothing but the Grothendieck group for the exact category of vector bundles. Similarly, the group of connected components for $\mathbb{K}^{\text {perf }}(Y)$ equals the Grothendieck group for the triangulated category of perfect complexes, which is defined in [Gro71, Exposé IV]. Therefore, we have the following corollary. 


\section{VECTOR BUNDLES AND GLOBAL RESOLUTIONS}

COROLlary 3.4. The group $K_{0}^{\text {perf }}(Y)$ is isomorphic to the free abelian group generated by isomorphism classes $[\mathcal{F}]$ of locally free $\mathcal{O}_{Y}$-modules of finite rank, modulo the relations $\left[\mathcal{F}^{\prime}\right]+\left[\mathcal{F}^{\prime \prime}\right]=[\mathcal{F}]$ for any exact sequence $0 \rightarrow \mathcal{F}^{\prime} \rightarrow \mathcal{F} \rightarrow \mathcal{F}^{\prime \prime} \rightarrow 0$.

Remark 3.5. Note that the proof of Corollary 2.5 works on every noetherian scheme $X$ for which any coherent $\mathcal{O}_{X}$-module is a quotient of a locally free $\mathcal{O}_{X}$-module of finite rank. Then, the same argument as in the proof of Proposition 3.2 shows that for such schemes the Waldhausen $K$-theory of perfect complexes coincides with the Quillen $K$-theory of vector bundles.

\section{Counterexamples}

Here we show that, on non-separated schemes, there are usually many coherent sheaves that are not quotients of locally free sheaves. Let $Z=\operatorname{Spec}(A)$ be an affine normal local noetherian scheme of dimension at least two, and $U \subset Z$ the complement of the closed point. Let $Y_{1}, Y_{2}$ be two copies of $Z$. The cocartesian diagram

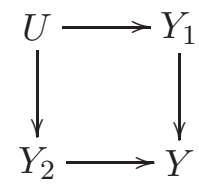

defines a non-separated normal noetherian scheme $Y$, endowed with an affine covering $Y=Y_{1} \cup Y_{2}$ with $Y_{1} \cap Y_{2}=U$.

Proposition 4.1. Every locally free $\mathcal{O}_{Y}$-module of finite rank is trivial.

Proof. Let $\mathfrak{U}$ be the open affine covering $Y=Y_{1} \cup Y_{2}$. We have $Z^{1}\left(\mathfrak{U}, \mathrm{GL}_{r}\right)=\Gamma\left(U, \mathrm{GL}_{r}\right)=\mathrm{GL}_{r}(A)$ and $C^{0}\left(\mathfrak{U}, \mathrm{GL}_{r}\right)=\prod_{i=1}^{2} \mathrm{GL}_{r}(A)$. This clearly implies that $H^{1}\left(\mathfrak{U}, \mathrm{GL}_{r}\right)=0$. Since any locally free $\mathcal{O}_{Y}$-module of finite rank becomes trivial on the local schemes $Y_{i}$, we also have $H^{1}\left(Y, \mathrm{GL}_{r}\right)=0$.

Proposition 4.2. There are coherent $\mathcal{O}_{Y}$-modules that are not quotients of locally free $\mathcal{O}_{Y}$-modules of finite rank.

Proof. Let $\mathcal{M}$ be a coherent $\mathcal{O}_{Y}$-module. Suppose there is an exact sequence

$$
\mathcal{O}_{Y}^{\oplus r} \longrightarrow \mathcal{O}_{Y}^{\oplus s} \longrightarrow \mathcal{M} \longrightarrow 0 .
$$

The map on the left is given by an $s \times r$-matrix with entries in $\Gamma\left(Y, \mathcal{O}_{Y}\right)=A$. This implies $\left.\left.\mathcal{M}\right|_{Y_{1}} \simeq \mathcal{M}\right|_{Y_{2}}$, where we use the canonical identifications $Y_{1}=Z=Y_{2}$. Now choose a coherent

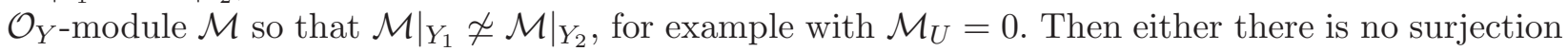
$\mathcal{O}_{Y}^{\oplus s} \rightarrow \mathcal{M}$, or there is such a surjection and its kernel $\mathcal{M}^{\prime}$ does not admit a surjection $\mathcal{O}_{Y}^{\oplus r} \rightarrow \mathcal{M}^{\prime}$. The statement now follows from Proposition 4.1.

\section{ACKNOWLEDGEMENT}

We thank the referee for his remarks and for pointing out some errors in the first version of the paper.

\section{REFERENCES}

ACG85 E. Arbarello, M. Cornalba, M. Griffiths and J. Harris, Geometry of algebraic curves, vol. I, Grundlehren Math. Wiss. 267 (Springer, New York, 1985).

BLP87 C. Bănică and J. Le Potier, Sur l'existence des fibrés vectoriels holomorphes sur les surfaces nonalgébriques, J. Reine Angew. Math. 378 (1987), 1-31. 


\section{VECTOR BUNDLES AND GLOBAL RESOLUTIONS}

Eis95 D. Eisenbud, Commutative algebra, Graduate Texts in Mathematics, vol. 150 (Springer, New York, 1995).

EG85 E. Evans and P. Griffith, Syzygies, London Mathematical Society Lecture Note Series, vol. 106 (Cambridge University Press, Cambridge, 1985).

GD70 A. Grothendieck and J. A. Dieudonné, Éléments de géométrie algébrique I: Le langage de schémas, Grundlehren der Mathematischen Wissenschaften, vol. 166 (Springer, Berlin, 1970).

Gro61 A. Grothendieck, Éléments de géométrie algébrique III: Étude cohomologique des faisceaux cohérents, Publ. Math. Inst. Hautes Étud. Sci. 11 (1961).

Gro65 A. Grothendieck, Éléments de géométrie algébrique IV: Étude locale des schémas et de morphismes de schémas, Publ. Math. Inst. Hautes Étud. Sci. 24 (1965).

Gro66 A. Grothendieck, Éléments de géométrie algébrique IV: Étude locale des schémas et de morphismes de schémas, Publ. Math. Inst. Hautes Étud. Sci. 28 (1966).

Gro67 A. Grothendieck, Éléments de géométrie algébrique IV: Étude locale des schémas et de morphismes de schémas, Publ. Math. Inst. Hautes Étud. Sci. 32 (1967).

Gro71 A. Grothendieck et al., Théorie des intersections et théorème de Riemann-Roch, Lecture Notes in Mathematics, vol. 225 (Springer, Berlin, 1971).

Har70 R. Hartshorne, Ample subvarieties of algebraic varieties, Lecture Notes in Mathematics, vol. 156 (Springer, Berlin, 1970).

Har77 R. Hartshorne, Algebraic geometry, Graduate Texts in Mathematics, vol. 52 (Springer, Berlin, 1977).

Har94 R. Hartshorne, Generalised divisors on Gorenstein schemes, K-Theory 8 (1994), 287-339.

Kle74 S. Kleiman, The transversality of a general translate, Compositio Math. 28 (1974), 287-297.

Lut93 W. Lütkebohmert, On compactification of schemes, Manuscr. Math. 80 (1993), 95-111.

Mar82 M. Maruyama, Elementary transformations in the theory of algebraic vector bundles, in Algebraic geometry, eds J. Aroca, R. Buchweitz and M. Giusti, Lecture Notes in Mathematics, vol. 961 (Springer, Berlin, 1982), 241-266.

Qui73 D. Quillen, Higher algebraic K-theory: I, in Algebraic K-theory I: Higher K-theories, ed. H. Bass, Lecture Notes in Mathematics, vol. 341 (Springer, Berlin 1973), 85-147.

Ray71 M. Raynaud and L. Gruson, Critères de platitude et de projectivité, Invent. Math. 13 (1971), 1-89.

Sch99 S. Schröer, On non-projective normal surfaces, Manuscr. Math. 100 (1999), 317-321.

Sch01 S. Schröer, There are enough Azumaya algebras on surfaces, Math. Ann. 321 (2001), 439-454.

Sch82 H.-W. Schuster, Locally free resolutions of coherent sheaves on surfaces, J. Reine Angew. Math. 337 (1982), 159-165.

Sch61 R. Schwarzenberger, Vector bundles on algebraic surfaces, Proc. London Math. Soc. 11 (1961), 601-622.

TT90 R. Thomason and T. Trobaugh, Higher algebraic K-theory of schemes and of derived categories, in The Grothendieck Festschrift III, eds P. Cartier et al., Progr. Math., vol. 88 (Birkhäuser, Boston, 1990), 247-435.

Voi01 C. Voisin, A counterexample to the Hodge conjecture for Kähler varieties, Preprint (2001), math.AG/0112247.

Stefan Schröer stefan.schroeer@uni-bayreuth.de

Mathematische Institut, Universität Bayreuth, D-95440, Bayreuth, Germany

Gabriele Vezzosi vezzosi@dma.unifi.it

Dipartimento di Matematica Applicata 'G. Sansone', Via di S. Marta 3, I-50139 Firenze, Italy 